\title{
Efficient QoS based mobile multicast using context transfer
}

\author{
Ilka Miloucheva ${ }^{1}$, Olaf Menzel $^{2}$, Karl Jonas ${ }^{3}$, Member IEEE, Rui L. Aguiar ${ }^{4}$, Member IEEE
}

\begin{abstract}
New challenging applications and scenarios for converged fixed and mobile Internet infrastructures, require efficient technologies adapting the multicast services for fixed IPv6 infrastructures to heterogeneous mobile environment.

This paper discusses architecture aimed to support QoS based mobile multicast in heterogeneous mobile IPv6 environment using context transfer between access routers for seamless handover of mobile multicast applications.

The architecture includes components for policy based QoS management of mobile multicast, reliable mobile multicast support at access routers and optimised handover based on learning of capabilities of access routers and networks.

Index Terms - Mobile communication, transport protocols
\end{abstract}

\section{INTRODUCTION}

Current communication developments are strongly focused on design concepts and architectures for mobile environments, using similar techniques and interfaces across heterogeneous technologies. This is becoming particularly relevant as mass-media multimedia entertainment services (such as TV) are now being targeted to also reach mobile devices.

The goal of this paper is the design of efficient mobile multicast services for mobile IPv6 environment using context transfer techniques for seamless handover. These services will be applied for new business scenarios including multimedia content delivery to mobile users, IP television, on-line gaming, software distribution, "rich" media, advertisements, video and audio on-demand.

In the EU IST project DAIDALOS, the context transfer is integrated for seamless handover and QoS support in heterogeneous mobile IPv6 architecture including different wireless technologies (WLAN, WIMAX, UMTS, Bluetooth) and broadcast media (DVB-T, DVB-H) [1],[2], [3]. Context transfer is deployed between access routers and QoS brokers processing state information, in order to achieve seamless handovers and QoS support.

The usage of context transfer for mobile multicast is aimed to support a complex set of tasks required for seamless continuation of multicast services considering the protocols and states of the mobile node's operating environment. The context transfer is independent on the mobility management

Ilka Miloucheva, Olaf Menzel and Karl Jonas are with SATCOM Advance Center of Fraunhofer Institute, Germany, Sankt Augustin, Germany, e-mail: \{ilka.miloucheva, olaf.menzel, karl.jonas\}@fokus.fraunhofer.de.

Rui L. Aguiar is with the Instituto de Telecomunicações, Portugal, e-mail: ruilaa@det.ua.pt.

Manuscript received December 12, 2005 and revised May 04, 2006. This paper was presented in part at the conference on Software, Telecommunications and Computer Networks (SOFTCOM) 2005

This work was supported by the EU IST project DAIDALOS. protocol, for instance Mobile IPv6 [4], so that it can be used in connection with the specific mobility scheme for seamless service continuation. Context-aware design for efficient mobile multicast services is based on components using a common infrastructure (with unified interfaces and welldefined functions). Especially, the overhead for mobile nodes should be reduced exploiting context managed by access routers, QoS brokers and other provisioning entities of the architecture.

The emphasis of the paper is on the policy based QoS management and transfer of contexts based on unified interfaces and mechanisms, aiming to enhance the QoS provision for mobile multicast nodes and improve the usage of network resources by multicast communication in heterogeneous network environment.

Multicast context transfer is combined with dynamic learning of access networks capabilities in Mobile IPv6 (MIPv6) [4], in order to optimise the selection of access router and network for multicast service continuation. Expected benefits of the proposed infrastructure are enhanced QoS, context-aware mobile networking, reliability and rapid re-establishment of mobile multicast services in case of handover and failure, as well as ubiquitous access to Internet content delivery services (from everywhere and at any time) by fixed or mobile users enabled with multiple devices and interfaces.

This paper is organised in following sections. Section 2 discusses the current state-of-the art of multicast mobility and context-aware mobile multicast techniques. Overview of the mobile multicast service requirements in DAIDALOS is given in section 3. Section 4 presents context transfer applied for IPv6 multicast protocols in mobile environment. Section 5 discusses support for enhanced mobile multicast including policy based QoS management, reliable mobile multicast and optimised handover based on learning of multicast capabilities of access networks. Section 6 concludes this paper.

\section{RELATED RESEARCH}

Techniques for seamless multicast handover and contextaware multicast transport are overviewed.

\section{A. Multicast Mobility in Heterogeneous Mobile IPv6 Environment}

\section{A.1 Introduction}

In converged fixed and mobile IP infrastructures, the goal is to adapt efficiently the standardised IETF Internet multicast solutions for group management and multicast routing to the particular mobility management scheme. 
IPv6 mobility support (MIPv6) proposed by IETF in RFC 3775[4] to address mobility management for IPv6 at network layer considering heterogeneous mobile network environment, is found to be not efficient for managing frequent mobility [27]. Efforts to improve mobile IPv6 [4] are aimed at minimizing the handover address configuration time and reducing the amount of signalling overhead required to re-route packets to the mobile node's new location.

In order to optimise mobility management, micro-mobility solutions [29] and protocols enhancing Mobile IPv6, such as Hierarchical Mobile IP [24] and Fast Handover [15], are proposed. The Host Identity Protocol (HIP) is aimed at enhanced terminal mobility management [11]. Recently, for optimisation of signalling overhead and efficient use of wireless resources, the network-based localized mobility (NetLMM) scheme is discussed [26].

Handover optimisation mechanisms aimed to be used with different mobility management schemes are Context Transfer [32], CARD protocol [18] and Media Independent PreAuthentication (MPA) [23].

In general, the seamless handover of mobile multicast services between different mobile access networks depends on the interaction of the particular mobility management scheme(s) with mechanisms aimed to provide quickly reestablishment of the multicast group membership and multicast delivery tree for the multicast node (sender or receiver) changing its point of attachment.

\section{A.2 Multicast Receiver mobility}

The multicast receiver mobility [5], [12] is addressing the quickly re-establishment of optimised multicast tree for the mobile multicast receivers attaching to new access network.

The approach of bi-directional tunnelling is aimed to adapt the IETF Internet multicast platform to mobile environment using a tunnel between Home Agents and mobile nodes for forwarding of multicast packets [4]. Due to the usage of tunnelling mechanisms, this technique is not scalable and wastes network resources involving point-to-point transport instead of multicast. On the other hand, in the remote subscription approach, the mobile node could join the multicast group on the next access network without waiting for the binding of its new care-of address. This reduces the delay for multicast service re-establishment [5]. However, during the handover process multicast packets could be lost. Another problem, in case of frequent handovers, is the increased overhead at the mobile nodes to re-join multicast groups.

The Fast Multicast Protocol for MIPv6, proposed in [14], is based on extension of MIPv6 for Fast Handovers [15]. It is aimed at establishment of the multicast listening context at the next access router before connecting of the mobile node to the new access network. This is achieved based on extension of the Fast Handovers messages with information, describing the active multicast groups of the mobile node, for which the new access router should establish multicast listening and routing context.

Alternatively mobile multicast could be also considered in the framework of Hierarchical Mobile IPv6 (H-MIPv6) [24]. The multicast packet forwarding is based on Mobility Anchor Points defined for the H-MIPv6 architectures [16].

An approach based on dynamically negotiated inter-agent handovers is presented in [17]. The agents are responsible for forwarding multicast data to the mobile node. The mobile nodes can optimize multicast routes and reduce the number of handoffs by selecting new multicast agents dynamically.

\section{A.3 Multicast Source mobility}

The focus of multicast source mobility is:

- Re-establishment of the multicast delivery tree, which is rooted at the source changing its point of attachment;

- Multicast route optimisation from the source to the multicast receivers.

New receivers have to wait for updating of the session announcements considering the new access network of the sender after the handover. Dependent on the used Any Source Multicast (ASM) or Source Specific Multicast (SSM) model, there are different approaches for multicast source mobility [5]. Considering ASM, the main approaches to handle source mobility in MIPv6 are:

- Bidirectional tunnelling of multicast streams from the source via Home Agent;

- Remote subscription with reconstruction of the multicast delivery tree;

- Agent based tree re-construction.

The usage of SSM for source mobility requires techniques for the re-establishment of a multicast session identified by $(\mathrm{S}, \mathrm{G})$, where $\mathrm{S}$ is the multicast source address and $\mathrm{G}$ the group multicast address. For this purpose, active subscription of the multicast receivers to the source address, when the mobile node moves to new access network, could be used, because the old multicast distribution tree invalidates. This intrinsically brings performance and scalability problems.

In [10], a tree morphing approach for IPv6 source mobility optimisation integrated to SSM routing is proposed. Further multicast tree optimisation approaches in the framework of mobile source movement are discussed in [20].

\section{A.4 Mobile multicast receivers with unidirectional links}

Besides the integration of mobile terminals (hosts) over wireless networks, the heterogeneous Internet environments can include mobile receivers with multicast services attached to unidirectional broadcast media (DVB-T and DVB-H) and satellites. Because multicast services depend on bidirectional connectivity (MLDv2, PIM-SM), the quickly establishment of tunnels into reverse (upstream) direction is required in order to support mobility of the mobile multicast receivers with unidirectional downstream connectivity.

In [21], possible solution for multicast mobility of nodes with unidirectional links based on usage of Link-Layer Tunnelling Mechanism for Unidirectional Links [22] in interaction with IPv6 Mobility protocol [4] and Fast Handovers [15] is discussed.

The approach is based on the unidirectional link feed description, which could be integrated in the "Options" Part of the Router Advertisement Message specified for MIPv6 [4].

In case of handover to unidirectional link, this allows simultaneously to the configuration of a new care-of-address of the mobile node to use the upstream tunnel to the feed for bidirectional communication. 


\section{A.5 Proactive multicast mobility}

In order to increase performance of moving multicast receivers and senders, proactive mobility techniques can be used. Proactive mobility could be based on different approaches for pre-allocation of states required for the multicast services of the mobile node after performing the handover. Thus, proactive mobility could include advance resource reservation, tree construction and routing optimisation, as well as application service installation for multicast support. Proactive QoS re-establishment is discussed in Next Steps in Signalling (NSIS) Working Group [38].

For multicast services with QoS guarantees and mission critical applications, reservation in advance can be used to reserve resources at the new access network at a future time, before the mobile node performs a handover to this access network. The multicast delivery tree and resources on the new access network could be established in advance dependent on the planned movement of the mobile sender or receiver [27].

Advance bandwidth reservation is a proactive mobility technique based on static and dynamic schemes [9]. In the static approach, bandwidth for mobile multicast services needs to be reserved at session start in all access networks, which the user will visit in the future. Dynamic schemes attempt to improve the bandwidth utilisation by trying to predict the next multicast access network, making the advance reservation dynamically before the times when the resources may be required.

\section{A.6 Summary}

Different multicast mobility challenges, considerations and techniques are summarised in figure :

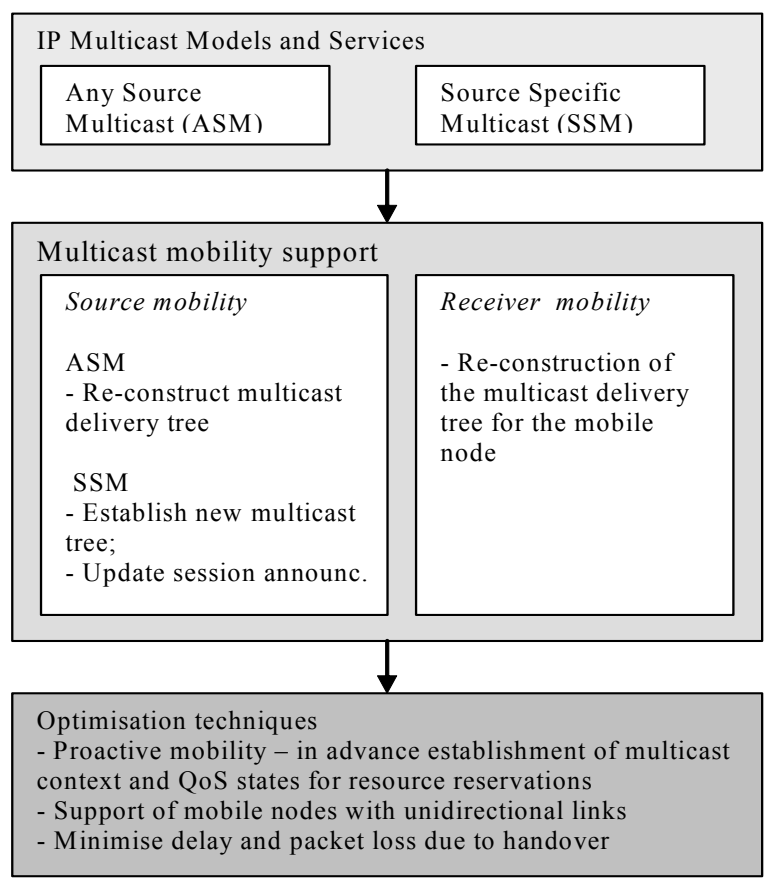

Figure 1: IP multicast mobility issues

\section{B. Context-Aware Multicast}

Context is a key issue in design of mobile services and technologies. The context-aware design of mobile multicast services considers groups of multicast receivers, resources, networking and operating environments. The management of different context types for protocols, services, user interactions and technologies is one important concept of the mobile networking today.

Context is an essential consideration of ubiquitous computing, where it is applied for rapid reestablishment of mobile services in case of handover and failure.

In user-centric architectures, the context could be used for optimised multicast access network selection in case of multiple access network interfaces considering user preferences and multicast application requirements. For instance, DVB-T can be preferred as efficient technology for multicast, when the handover choice can be done between multiple heterogeneous access technologies.

In [25], the context is defined through four dimensions, which complement and interact with each other:

- System context focussed on the technological issues, such as devices and applications;

- Infrastructure context concerning the connections between the devices that comprise the system and the information accuracy due to connectivity;

- Domain context related to the specific situated interaction that takes place in the specific domain;

- Physical context addressing the relation of the system to the physical environment and devices (for instance location, mobility, population).

For mobile multicast, the infrastructure context can include considerations of capabilities of access networks and installations of multicast sources, while the system context can be focussed on available protocols and services for multicast routing and group communication.

When the mobile node moves, its contexts at the previous and next access network are updated based on interactions of the peer-to-peer context transfer entities. This is exploited in multiple mechanisms for mobility speed-off, information caching, predictive preparation of handover, and adequate selection of new access point [28], [29], [30], [31]. All of these issues benefit from context transferral and from context exposure previous to handover, thus leading to a faster, smoother handover process.

Most of these context-aware design challenges are leading to the design of the Context Transfer Protocol, developed by the Seamoby IETF Working Group [32] as an Experimental protocol for enhancement of mobility management schemes. Context transfer is a technology used to achieve mobility based on different kind of contexts, such as security, accounting, QoS provisioning [33], [34]. These kinds of contexts are processed by peer-to-peer entities, which could be access routers, but also servers and QoS brokers.

In order to minimize the needed authentication operations during the handover and quick services re-establishment, context transfer is offering a common technology for unified state updates used also for exchange of mobile node's accounting and security information between access routers. 
Context-aware technologies and approaches, which could be applied to mobile multicast service development, are summarised in figure 2:

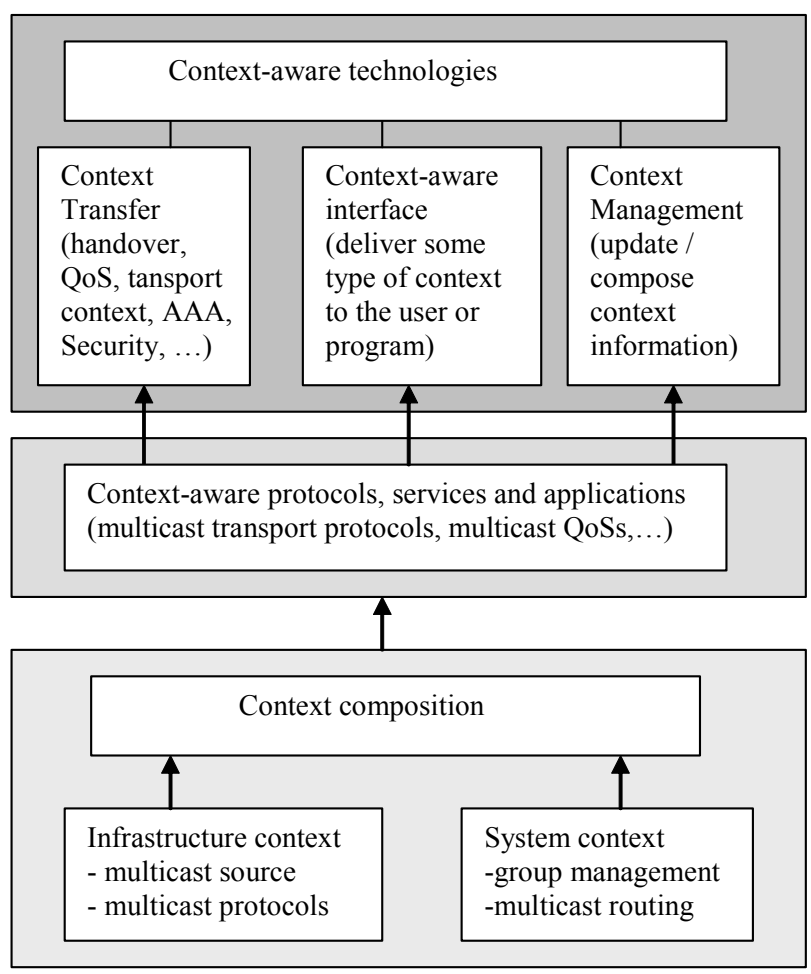

Figure 2: Context- aware design approaches applied to mobile multicast

\section{Mobile Multicast Services}

DAIDALOS architecture is aimed to provide an advanced Service Provisioning Platform for QoS-based mobile multimedia applications in heterogeneous IPv6 environment [1], [2], [3].

The focus is seamless handover between different kinds of wireless technologies (WiFi, WiMAX, TD-CDMA, and Bluetooth) and broadcast media (DVB-T, DVB-H). Components for QoS provisioning and resource reservation, performance measurement, accounting, authorisation and security handling are included to consider convergent fixed and mobile Internet infrsatructures

A distributed Service Provisioning environment is deployed to manage and control the Quality of unicast and multicast transport to mobile receivers. It is based on QoS, Authentication, Authorisation, Auditing, Accounting, Charging (A4C), and Multimedia sub-systems.

QoS aspects are clearly important for the mobile multicast services. Such are:

- Management, optimisation and planning of transport resources for multicast applications, especially on the critically limited access networks, using distributed QoS brokers;

- Seamless handover based on cross-network mapping of QoS parameters between different wireless networks (TD-CDMA, WLAN, WIMAX) and broadcast media (DVB);

- Flexible application interfaces for negotiation and control of scalable application QoS, supporting also
Automated configuration and parameterisation of scalable QoS requirements of multicast applications

The networking architecture for QoS based multicast transport to mobile users is shown in figure 3:

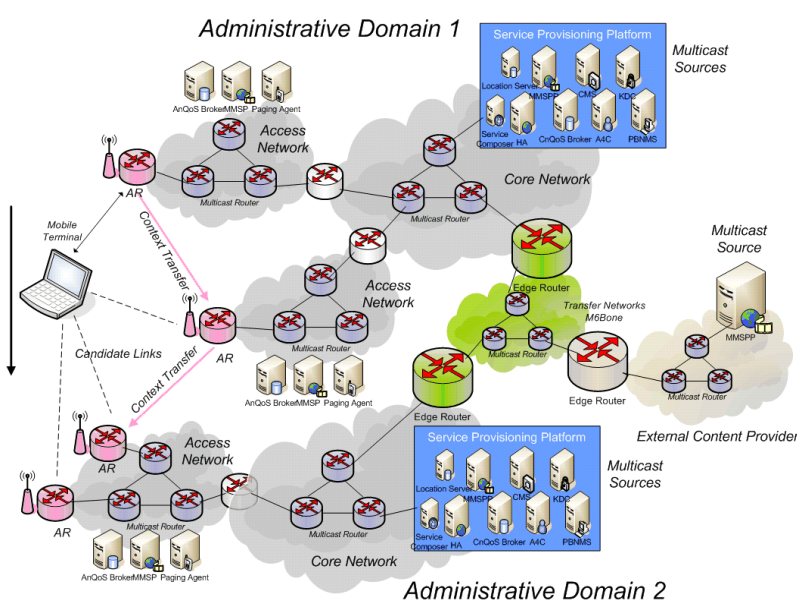

Figure 3: Networking architecture for QoS based multicast transport

Mobile multicast services supported by the DAIDALOS multicast platform include:

- Streaming multicast for continuous media transfer (audio, video, text) with time synchronisation;

- $\quad$ Reliable multicast transport (binary file download);

- Carousel data delivery (synchronised media and reliable file delivery for later display).

The key issue of the context transfer used for mobility in DAIDALOS is the context-oriented platform for adaptation of different Internet services to the mobile environment.

This allows an efficient mobile architecture based on service interactions using common context management and transfer functions.

Mobile multicast applications aimed at simultaneous delivery of multimedia content to groups of users in heterogeneous mobile infrastructure are designed in contextaware manner adapting to the network, application and operating environment.

\section{Multicast Context Transfer}

Context transfer is a technology applied between peer-topeer entities (access routers, QoS brokers, content servers) exchanging states (control data) related to the mobile node's active protocols and services, when the node changes its point of attachment [33]. Context transfer is used to achieve mobility based on different kind of contexts, such as QoS provisioning [30], authentication, authorisation, auditing, accounting and charging [34], security [35], and accounting [36], processed by peer-to-peer entities.

When the mobile node with a multicast service moves from one access network to another, contexts of different kind are re-established. In particular, contexts could describe:

- Multicast protocol listening and routing information;

- QoS parameters;

- Content delivery options; 
- Resource reservation states;

- Identity, accounting, authorization and security information;

- Metering data of resource usage.

Context transfer supports the replication of state information about the different kinds of contexts concerning the mobile node, allowing context-aware protocols and interfaces to be activated with less delay and less signalling overhead. Context transfer could be also used for enforcement of QoS management policies in heterogeneous mobile environment, for instance load balancing and flow distribution to different access routers, assignment of mobile nodes to optimal access router considering traffic policies, dispatching of mobile traffic to distinct paths and access networks.

The Context Transfer Protocol (CXTP), developed by the Seamoby IETF Working Group [32], aims to provide general mechanisms for the exchange of context data for moving mobile nodes between access routers. It defines control messages and procedures for initiation and authorisation of context transfer between access routers, as well as for transferring control data states related to the mobile users and their services before, during and after the handover. As result of the context protocol message exchange, context information is stored at the target entity for delivery to the specific module.

The multicast context transfer of a mobile node is aimed to re-establish the listening and routing states at the new access router for the active multicast groups and sources required by the services of the mobile node attaching to the new network.

In DAIDALOS, the multicast context transfer for seamless handover in heterogeneous IPv6 environment is combined with standardised by IETF multicast group management and routing solutions, such as:

- Multicast Listener Discovery version 2 - MLDv2 [7] protocol for multicast group management supporting also SSM;

- $\quad$ Protocol Independent Multicast - Sparse Mode (PIM$\mathrm{SM}$ ) [8], which is currently the most widely used protocol for multicast routing used also in inter-domain infrastructures.

To support mobile multicast in such protocol environment, multicast context transfer block (M-CTB) is specified [19] including the feature data, i.e. multicast addresses and other information required for the multicast services of the moving mobile node.

M-CTB consists of a list of multicast group records, one for each group the mobile node is interested in. Each multicast group record describes the mobile node's multicast filter for the specified multicast group, multicast source list and additional optional information for access, authorization group and optimization hints.

Using M-CTB data, MLDv2 is responsible to update its states at the next access router based on feeding of this information into the active multicast routing protocol (PIM$\mathrm{SM}$ ), so the multicast distribution trees may be built to serve the listeners in a particular interface.

MLDv2 protocol states according to the specification [7] provide aggregated information on the active listeners for a particular multicast group and interfaces. Based on this, the multicast access router (PIM-SM) knows that "at least one" mobile node is listening to a particular multicast address and source at given interface. The MLDv2 context for mobile nodes at multicast access routers is built based on the standardised MLDv2 protocol states per interface and group [7] enhanced with context information for the mobile node's active multicast group and sources, as described in [19].

The context transfer for multicast services in the handover preparation phase could be initiated by the mobile terminal or access network (previous or next access router, to which the mobile node is connected).

There are different scenarios and management policies for handovers initiated by network and mobile node.

Network initiated handovers are used in scenarios aimed at better utilization of network resources. In user-centric mobile infrastructures, QoS policies can specify rules for intelligent access router selection and selection of service provider offerings based on user profile and application preferences. Example scenarios for enforcement of network initiated handover using policies are:

- Change of the access routers for a mobile node based on business and strategic goals;

- Movement to a new access network due to traffic overload;

- $\quad$ Load balancing between different access routers and access networks;

- Selection of most appropriate access router and network for mobile node with specific services (content delivery, Video-on-demand, caching requirements, server connection);

- Traffic control and engineering between different access routers for optimised network resource usage;

- Optimising content delivery to mobile nodes using specific routes and access routers.

Particularly, in case of handover of mobile nodes with multicast services, the network initiated handover could be aimed at reducing the overhead for multicast routing based on selection of a new access router with already established multicast routes to multicast groups required by the mobile node. The network mobility management module can collect information for the activation of the handover of the mobile node using different kinds of measurement and monitoring data bases:

- Data link layer measurements obtaining performance parameters of wireless networks and broadcast media;

- Traffic measurements describing access network connections;

- $\quad$ Access router traffic load patterns;

- Traffic predictions based on monitoring of the mobile traffic flows;

- Resource monitoring and prediction data, as well as requests for advance resource reservations on network connections [32].

CXTP defines two scenarios for the network controlled handover (figure 4):

- Predictive - started by the previous Access Router (pAR);

- $\quad$ Reactive - started by the next Access Router (nAR). 


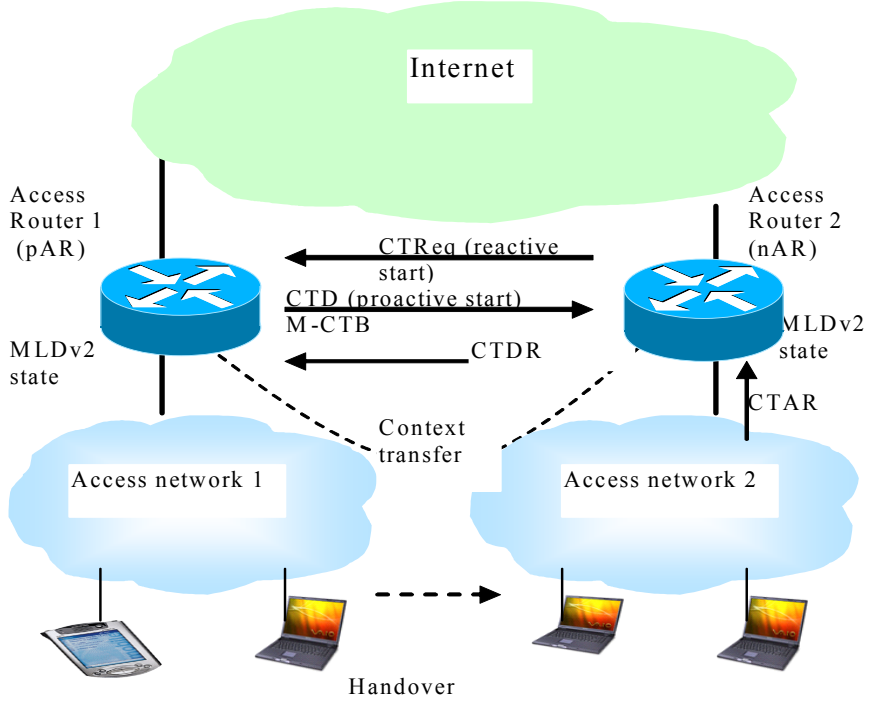

Figure 4: Predictive and reactive network controlled multicast context transfer

In predictive network controlled scenario, pAR initiates the context transfer with the Context Transfer Data (CTD) message including the M-CTB structure with the mobile node's active multicast groups. M-CTB is built in pAR with input from the MLDv2 entity.

In reactive scenario, this message is returned as answer to the CTReq. After receiving CTD, the nAR schedules it to the MLDv2 entity, in order to establish an individual node context for the used multicast groups and to update the aggregated MLDv2 listening states. This change in the aggregated state will then trigger the multicast routing protocol (PIM-SM) to build at nAR the distribution trees for each of the multicast groups. The CTDR message is sent optionally by nAR to pAR as response of CTD.

The predictive and reactive scenario could be used to provide different management policies. For example, the predictive scenario could be used, when the pAR has access to network topology and want to provide traffic balancing, if it experiences an overload situation. The reactive scenario could be applied by the nAR in order to support seamless handover, when the user profile and preferences of the mobile node are known in advance.

In order to provide efficient mobile multicast based on network-controlled handover, access router federation infrastructures could be considered in the future [40]

In the mobile node controlled handover, the mobile node sends a CTAR message either to the pAR and nAR in order to activate the context transfer. After this, the context transfer is executed as already described.

The mobile node initiated handover could be enforced by the mobile node considering its individual QoS experience, as for instance QoS disturbance, "ping-pong" effects, lost of connectivity, cost preference and reliability.

The multicast context transfer is independent from the mobility scheme and can be done based on interactions with Mobile IPv6 (MIPv6) [4], Fast Handovers [15], and/or Candidate Access Router Discovery (CARD) protocol [18], as it is discussed in [19].

\section{Support Of Mobile Multicast}

\section{A. Policy Based Multicast QoS Management}

In DAIDALOS, the management of QoS provisioning for multicast delivery is based on hierarchically distributed QoS brokers at access, core, and inter-domain level [37].

At the top of this hierarchy the Core QoS Broker is responsible for Inter-Domain and Inter-Access Network Resources provisioning based on Differentiated Service (DS) architecture, while the Access Network (AN) QoS Brokers provide flow based QoS to the mobile terminals using signalling mechanisms for Integrated Service support.

Part of the QoS architecture is the Multicast Manager, which handles the reservation of multicast resources and performs tree optimizations for mobile nodes with multicast services.

Context transfer and QoS signalling between brokers are used to localize RSVP reservation state repairs of multicast trees in mobile environment, when a mobile node crosses QoS Broker domains. The QoS Brokers decisions are taken based on Service Level Agreements and policies considering performance measurements from routers and network probes.

The core QoS brokers collect measurements about multicast routing paths, especially:

- Network performance metrics of routing paths connecting the multicast access routers with the service provisioning platform;

- Availability and load characteristics of core routers belonging to the multicast delivery tree.

Communication between core and AN QoS Brokers follows specific policy based mechanisms, described by a Policy Decision Point - Policy Enforcement Point (PDP-PEP) model, while communication between AN QoS Brokers uses a peer-to-peer model.

Policies allow the operator to control dynamically the QoS provisioning for mobile multicast users [6]. QoS policy examples for mobile multicast are:

- Regulation of access to the QoS considering layered multicast QoS model and user profiles;

- Enhancement of the QoS for particular applications based on restriction of resource usage at certain time;

- Optimised handover decisions using specific criteria for selection of appropriate access network to reduce costs and avoid overload;

- Usage of specific routes for specific multicast groups, services and applications;

- Multicast service redirection, when in case of a failure of access router specific multicast access router should be used to continue the multicast services.

In the QoS management scenario, illustrated in figure 5, Core and AN QoS Brokers are shown, which interact with performance measurement data base in order to plan efficiently the network resource usage by mobile multicast applications. 


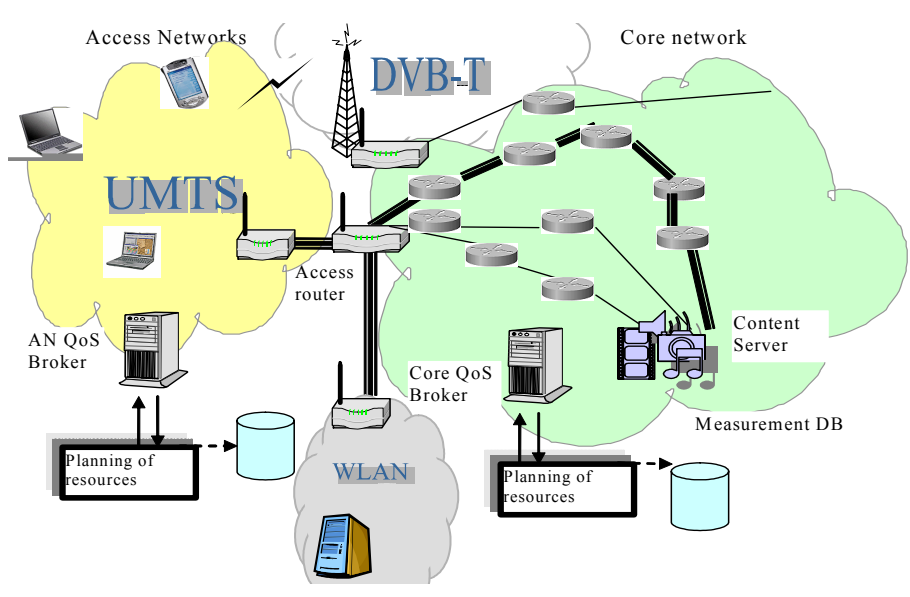

Figure 5: QoS broker architecture for management and planning of resources for enhanced multicast

Policy based QoS management is used to control the multicast distribution from a given server to the multicast users. Policies restrict the multicast transfer for specific services based on resource planning strategies and prediction of mobile user's behaviour for content delivery.

In the shown scenario, the multicast transfer from the content server to the user group with mobile receivers is performed via specific access router(s) and networks (UMTS and WLAN).

\section{B. Reliable Multicast Support at Access Routers}

In [13], enhancement of the multicast architecture of DAIDALOS for reliable content delivery scenarios is described. These services will be used for one-to-many file downloads and content distribution scenarios.

For enhanced reliability, access routers are used to provide local retransmission strategies dependent on the access network (wireless, broadcast media). The received data from the server is cached at the access routers, which transmit it reliably to the mobile multicast nodes attached to them.

The local retransmission schemes are configured in end-toend tree-based reliable multicast transport (see Figure 6).

The end-to-end multicast for one-to-many content delivery consists of schemes for:

(1) Reliable transfer from the server(s) to access router(s);

(2) Caching at access routers and reliable multicast to attached mobile nodes.

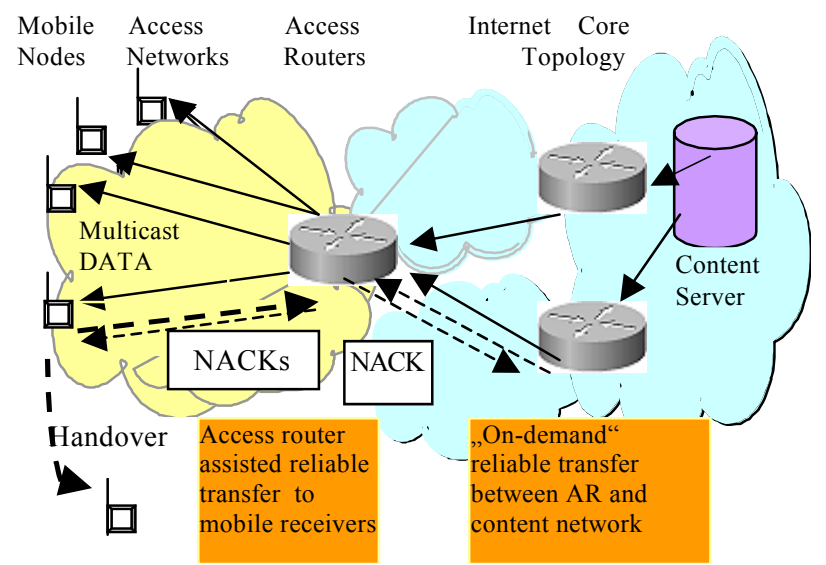

Figure 6: Access router supported of multicast reliable transport
NACK-repair requests are sent by the mobile nodes to the actual access router, which filters them and retransmits lost data based on the established context for reliable multicast transport.

The benefit of access router assistance is that in case of handover, using context transfer between the access routers, the missing data could be sent to the new access router. Thus, the reliable multicast transport could be continued without degradation of throughput due to packet losses experienced by mobile nodes in case of handovers.

Caching at access routers is important to support further mobile multicast services and scenarios, such as asynchronous multicast (when mobile nodes are joining the multicast group after session establishment), multicast streaming service combined with recording (reliable storage) and resilient multicast (service based on waiting for the retransmissions within a given time interval).

\section{Optimised Handover for Mobile Nodes with Multiple Network Interfaces}

In user centric infrastructures, a mobile node can be configured with multiple links to access networks supporting multicast (WLAN, broadcast media, UMTS, WIMAX).

The availability of multiple links can be used for optimisation of mobile multicast, in particular to provide ubiquitous, cost-efficient, permanent and fault-tolerant access to Internet services.

Currently, in MIPv6 environment, the support of mobile nodes with multiple network interfaces used simultaneously (multi-homed mobile node architecture) is focus of research and standardisation (IETF Monami6 Working Group) [39].

One scenario for enhanced multicast based on multiple interfaces is optimised multicast access router selection. In order to support efficient handover or to optimise network resource utilisation and user costs, the new multicast access router for the mobile multicast session could be selected according specific policies and optimisation criteria.

As example, a mobile multicast node, which receives a multicast video delivery on WLAN, can perform handover to another access network, such as DVB-T or DVB-H, which are more cost-efficient.

The Candidate Access Router Discovery (CARD) protocol [18] is a technology, which can be used to learn characteristics of the multicast access routers for optimised new access router and network selection.

Handover decisions for heterogeneous environment can be based on attributes of candidate access routers and networks provided by CARD, such as:

- Multicast group and sources (servers), for which routing context (multicast distribution tree) is established at the candidate access router;

- Multicast services supported at the router;

- Cost for bandwidth usage at given access network;

- Bandwidth reservation for the multicast session;

- QoS provision and traffic load.

Interactions of CARD protocol, QoS management system, access routers and mobile nodes, are shown in Figure 7: 


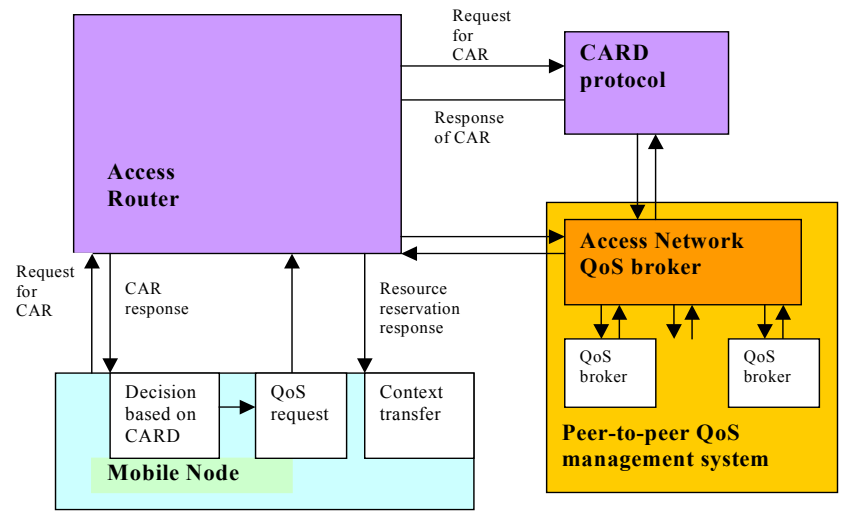

Figure 7: Interactions for optimized multicast access router selection

The CARD Daemon asks the AN QoS broker of the access network for attributes of possible access routers considering specific content delivery services required by the mobile node. The QoS management system provides the data to the CARD protocol in form of required capabilities and attributes for possible candidate access routers.

Based on these characteristics and the policies stored in the system, the mobile node or the network selects the optimal multicast access router and network for the handover of the multicast session.

\section{CONCLUSION}

Architecture for efficient mobile multicast transport in converged fixed / mobile user-centric environment was discussed.

This architecture is adequate to streaming mass-media services in an integrated heterogeneous IPv6 mobile infrastructure, supporting both bi-directional and unidirectional technologies. It was mostly developed under the framework of the DAIDALOS project. IETF-based protocols, such as Context Transfer and CARD, are deployed and integrated in the framework of DAIDALOS in order to enhance mobile multicast service provision.

Further mechanisms for efficient mobile multicast based on advance pro-active resource reservation and QoS policy control are currently the focus of research and development.

\section{ACKNOWLEDGMENT}

The work is based on of the mobile multicast architecture developed within the EU IST project DAIDALOS. Thanks are due to the European commission supporting this project and to all the partners that contributed to this work, and in particular to our colleagues inside Fraunhofer, Instituto de Telecomunicações and Portugal Telecom Inovação.

\section{REFERENCES}

[1] DAIDALOS - Designing Advanced Interfaces for the Delivery and Administration of Location independent Optimised personal Services, www.ist-daidalos.org

[2] R. Aguiar, D. Bijwaard, B. Farshichian, K. Jonas, A. Sarma: "Pervasive Services for Next Generation Heterogeneous Networks", World Telecommunications Congress 2006, May 2006
[3] I. Miloucheva, H. J. Einsiedler, D. Gomes, K. Jonas: "QoS based multicast architecture for heterogeneous mobile IPv6 environment", 13th International Conference on Telecommunications, 9-12 May 2006

[4] D. Johnson, C. Perkins, J. Arkko: "Mobility Support in IPv6", RFC 3775, June 2004

[5] I. Romdhani, M. Kellil, H-Y. Lach, A. Bouabdallah, H. Bettahar: "IP Mobile Multicast: Challenges and Solutions", IEEE Communications Surveys \& Tutorials, 2004

[6] H. Zheng, M. Greis: "Ongoing Research on QoS Policy Control Schemes in Mobile Networks", In Mobile Networks and Applications 9, p. 235-241, 2004

[7] R. Vida, L. Costa: "Multicast Listener Discovery Version 2 (MLDv2) for IPv6", RFC 3810, June 2004

[8] D. Estrin et al, "Protocol Independent Multicast - Sparse Mode - Protocol Specification", RFC 2362, June 1998

[9] J. Ye, S. Papavassiliou, G. Anastasi, A. Puliafito, "Strategies for Dynamic Management of the QoS of Mobile Users in Wireless Networks through Software Agents", IEEE, 2002

[10] T.C. Schmidt, M. Wählisch: "A First Performance Analysis of the Tree Morphing Approach to IPv6 Source Mobility in Source Specific Multicast Routing, Proceedings of the $5^{\text {th }}$ International Conference on Networking, 2006

[11] K. Moskowitz, P. Nikander: "Host Identity Protocol (HIP) Architecture", RFC 4423, Mai 2006

[12] M. Vieth, O. Menzel, K. Jonas, I.Miloucheva, J.F. Placido, H. Santos, E. Guainella: "Learning for Efficient Handover of QoS Based Multicast Services", CITSA Conference, Orlando, USA, July 2005

[13] J. Mahnke, N. Reyes, I. Miloucheva: "Protocol Mechanisms for Reliable Multicast in Mobile IPv6 Environment", IPS-MoMe Workshop, Salzburg, February 2006

[14] K. Suh, D-H. Kwon, Y-J. Suh, Y. Park: "Fast Multicast Protocol for Mobile Ipv6 in the fast handovers environment", Internet Draft, January 2004

[15] R. Koodli: "Fast Handovers for Mobile IPv6", RFC 4068, July 2005

[16] T.C. Schmidt, M. Wählisch: "Performance analysis of multicast mobility in a Hierarchical Mobile IP Proxy Environment", TERENA Networking Conference, Rhodos, Greece, June 2004

[17] H. Zhang, B. Zhang, B. Shen: "An efficient dynamic multicast agent approach for mobile IPv6 multicast", Academic Open Internet Journal, Vol. 17, 2006

[18] M. Liebsch, A. Singh, H. Chaskar, D. Funato, E. Shim: "Candidate Access Router Discovery (CARD)", RFC 4066, July 2005

[19] H. Santos, R. Aguiar, I. Miloucheva, K. Jonas: "Multicast Context Transfer for Mobile IPv6 Receivers", IETF Draft, February 2006

[20] H. Gossain, S. Kamat, D. P. Agrawal: "A Framework for Handling Multicast Source Movement over Mobile IP”, IEEE ICC, Conference, April 2002

[21] O. Menzel, D. Wagner, I. Miloucheva: "Support of mobile nodes with unidirectional links in MIPv6", Internet Draft, April 2006

[22] E. Duros, W. Dabbous, H. Izumiyama, N. Fujii, Y.Zhang: "A Link-Layer Tunnelling Mechanism for Unidirectional Links", RFC 3077, March 2001

[23] A. Dutta, V. Fajardo, Y. Ohba, K. Taniuchi, H. Schulzrinne: "A Framework of Media-Independent Pre-Authentication (MPA)", MOBOPTS, Internet Draft, March 2006

[24] H. Soliman, C. Castelluccia, K. ElMalki, and L. Bellier: "Hierarchical Mobile IPv6 Mobility Management (HMIPv6)", RFC 4140, August 2005. 
[25] G. Chen, D. Kotz: "Context Aggregation and Dissemination in Ubiquitous Computing Systems“. In Workshop on Mobile Computing Systems \& Application, 2002

[26] G. Giaretta, K. Leung, M. Liebsch, P. Roberts, K. Nishida, H. Yokota, M. Parthasarathy, H. Levkowetz: "NetLMM Protocol", Internet Draft IETF NETLMM Working Group, June 19, 2006

[27] N. Van den Wijngaert, C. Blondia: „“A predictive Low Latency Handover Scheme for Mobile IP“, Second International Conference on Mobile Computing and Ubiquitous Networking, ICMU, Japan, 2005

[28] A. Mishra, M. Shin, W. A. Arbaush.: "Context caching using neighbor graphs for fast handoffs in a wireless network", Twenty-third Annual Joint Conference of the IEEE Computer and Communications Societies, INFOCOM, 7-11 March 2004

[29] J. M. Oyoqui, J.A. Garcia-Macias: "Context transfer for seamless micro-mobility", Proceedings of the Fourth Mexican International Conference on Computer Science, ENC, page(s):291 - 297, 2003

[30] S. Pack, H. Jung, T. Kwon, Y. Choi: “A selective neighbor caching scheme for fast handoff in IEEE 802.11 wireless networks", IEEE International Conference on Communications, ICC, May 2005

[31] S. Leggio, J. Manner, and K Raatikainen: "Achieving Seamless Mobility in IP-Based Radio Access Networks" IEEE Wireless Communications, Volume 12, Issue 2, p.54- 59, Feb. 2005

[32] J. Loughney, M. Nakhjiri, C. Perkins, R. Koodli: "Context Transfer Protocol (CXTP)", RFC 4067, July 2005

[33] J. Kempf: "Problem Description: Reasons for Performing Context Transfers between Nodes in an IP Access Network", RFC 3374, September 2002

[34] M. Georgiades, N. Akhtar, C. Politis, T. Tafazolli: "AAA Context Transfer for seamless and secure multimedia services over All-IP networks", 5th European Wireless Conference, Barcelona, February 2004

[35] Y. Ouyang; C. Chu: "A secure context transfer scheme for integration of UMTS and 802.11 WLANs" IEEE International Conference on Networking, Sensing and Control, March 2004

[36] C. Politis, K. A. Chew, N. Akhtar, M. Georgiades, R. Tafazolli, T. Dagiuklas: "Hybrid multilayer mobility management with AAA context transfer capabilities for all-IP networks" IEEE Wireless Communications, Volume 11, Issue 4, p. $76-88$, Aug. 2004

[37] R. Prior, S.Sargento, D.Gomes, R.Aguilar: "Heterogeneous Signalling Framework for End-to-end QoS support in NGN", International Conference on System Sciences, QoS Track, Hawaii, January 2005

[38] F. Tommasi, S. Molendini, A Tricco, E. Scialpi: "Semiproactive QoS Re-establishment”, IETF NSIS Working Group, Internet Draft, Work in Progress, February 2006

[39] N. Montavont, R. Wakikawa, T. Ernst, C. Ng, K. Kuladinithi: "Analysis of Multihoming in Mobile IPv6", IETF MONAMI6 Working Group, Internet-Draft, draft-ietf-monami6-mipv6analysis-00.txt (work in progress), February 20, 2006

[40] J. Guan, Z. Sun: "Requirements for Router Federation", draftguan-router-federation-00.txt, Internet Draft, March 2006

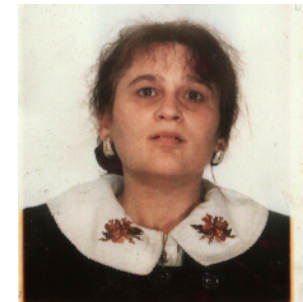

Dr. Ilka Miloucheva holds the doctor of engineering degree in informatics with suma cum laude from the Technical University Dresden, Germany. She worked as scientific fellow at Technical University Berlin (Germany) and Salzburg Research (Austria) participating in different European projects in the area of high speed communication, multimedia protocols and measurement architectures. She initiated international workshops: PROMS (Protocols on Multimedia Systems) and IPS (Inter-domain Performance and Simulation), and participated in the programming committee of different conferences IPCCC, ICECS, NE2WAN and CITSA. She was technical manager of EU IST INTERMON project and is in the management committee of COST 290 Action "Traffic and QoS management in wireless networks" on behalf of Salzburg Research. Since 2004 she joined SATCOM Centre of Fraunhofer Institute and University of Applied Sciences Bonn-Rhein-Sieg, currently involved in the EU IST projects DAIDALOS and NETQOS.

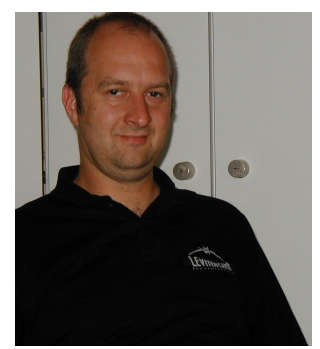

Dipl. Ing. Olaf Menzel hold a diploma of engineering degree in computer science from the University of Applied Sciences (TFH) in Berlin. In 1998 he joined the German National Research Centre for Information Technology (GMD) for writing his diploma thesis in satellite communications. In 1999 he continued at GMD with many projects in the satellite and next generation IP network realm with the special focus on DVB IPv6 integration, IP security, IP mobility support and IP Multicast handover support for unidirectional links.

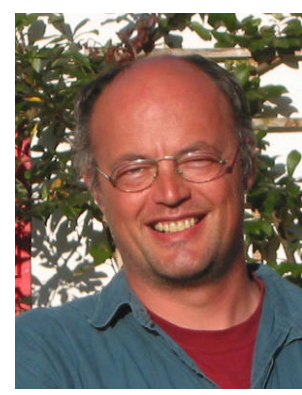

Prof. Dr. Karl Jonas holds a diploma and a doctor of engineering degree in computer science from the Technical University of Berlin. In 1994 he joined the German National Research Centre for Information Technology (GMD), installing a research group for multimedia Internet services. In

1998 he joined the NEC research laboratory in Heidelberg, where his

activities focused on multimedia applications, multipoint services and mobility support for real-time applications. Since 2001 he is a professor for multimedia communication at the University for Applied Science in Bonn-Rhein-Sieg, and head of the Fraunhofer Fokus competence center for advanced satellite communication.

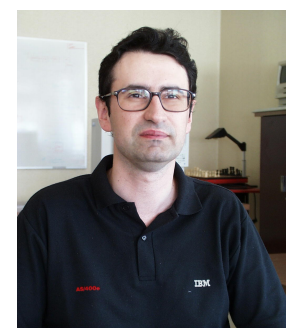

Prof. Dr. Rui L. Aguiar holds a diploma and a masters on Electronics and Telecommunications, and a $\mathrm{PhD}$ on Electrotechnic from the Universidade de Aveiro, where he is a Professor since 2001. He is coordinating a group on heterogeneous networking inside Instituto de Telecomunicações, Aveiro center. His participation in European-cooperative research is extensive, and he is currently Chief Architect for the DAIDALOS project. His current interests are on high-performance heterogeneous systems and circuits, and has more than 150 published papers in those areas. He has been chair of several conferences, and is currently TPC Co-Chair for ISCC2007. 